\title{
Procédure à suivre lors de rappels d'implants cardiaux
}

\section{Jürg Fuhrer}

Président du et au nom du comité du groupe de travail «Stimulation cardiaque et électrophysiologie» de la Société suisse de cardiologie
Correspondance:

Dr Jürg Fuhrer

Universitätsklinik und -poliklinik

für Kardiologie

Departement Herz und Gefässe

Inselspital

CH-3010 Berne

Tél. 0316329654

Fax 0316321414

juerg.fuhrer@insel.ch

\section{Introduction}

La publication de rapports sur des implants rappelés par les fabricants pour cause de dysfonctionnement potentiel a fortement augmenté ces dernières années. Ceci a suscité chez de nombreux patients, leurs proches, leurs médecins et d'autres milieux concernés, un débat quant à la façon de procéder dans une telle situation.

Les fabricants et les utilisateurs concernés sont soumis à des dispositions juridiques précises, outre les obligations habituelles visant à minimiser le risque chez les patients implantés. L'article 3 de la loi sur les produits thérapeutiques s'applique ici par analogie: «Devoir de diligence: Quiconque effectue une opération en rapport avec des produits thérapeutiques est tenu de prendre toutes les mesures requises par l'état de la science et de la technique afin de ne pas mettre en danger la santé de l'être humain et des animaux.»

Il n'est pas toujours facile d'agir correctement lors de rappels d'implants pour cause de dysfonctionnement potentiel. Le présent commentaire a pour but de fournir des recommandations générales importantes sur la manière de procéder dans les cas particuliers lors de tels rappels par les fabricants. Il a été mis au point par le comité du groupe de travail «Stimulation cardiaque et électrophysiologie» de la Société suisse de cardiologie en collaboration avec l'autorité suisse qui délivre les autorisations (Swissmedic), la Société suisse de médecine légale, la Fédération des associations suisses du commerce et de l'industrie de la technologie médicale (FASMED) et toutes les autres firmes actives dans ce domaine en Suisse. Le texte ci-après est le reflet d'un consensus obtenu avec tous les participants. Il est publié sous la responsabilité de la Société suisse de cardiologie, de santésuisse, du service juridique de la FMH et du Dachverband Schweizerischer Patientenstellen (DVSP), (cf. appendice).

Notre commentaire est conçu pour venir en aide dans des situations pouvant s'avérer très complexes. Quiconque observe nos recommandations remplira en principe le devoir de dili- gence. Ce commentaire peut toutefois être incomplet dans certains cas, et il n'est pas exclu que des mesures supplémentaires ou autres soient nécessaires selon la situation. En conséquence, aucune prétention juridique ou en responsabilité quelconque ne peut être formulée, en raison de ce texte ou en relation avec celui-ci, à l'encontre du comité du groupe de travail, des fabricants ou des autres parties susmentionnées.

\section{Définitions}

Le terme «rappel» utilisé dans le présent commentaire correspond aux définitions données par les autorités européennes dans leurs lignes directrices sur la matériovigilance. Les recommandations officielles du fabricant («dear doctor letter») sont, quant à elles, désignées sous le nom de «fiche d'avertissement» («advisory notice»). La Suisse a inclus les directives européennes dans son Ordonnance sur les dispositifs médicaux et elle est tenue de les respecter en vertu des accords bilatéraux. Voici brièvement les définitions courantes.

\section{Définitions européennes}

(MEDDEV 2.12/1, révisé le 4 avril 2001: Lignes directrices sur un système de vigilance [«Guidelines on a medical devices vigilance system»], éditées par la Commission européenne, Entreprise et Industrie, direction générale):

Rappel («recall»): (1) En cas de risque mortel ou de détérioration sérieuse de l'état de santé, (2) en cas de retour au fournisseur d'un dispositif médical, (3) en cas de modification du dispositif par le fournisseur sur le site d'installation, (4) en cas d'échange ou de destruction du dispositif selon les instructions figurant sur la fiche d'avertissement. Fiche d'avertissement («advisory notice»): notice diffusée pour fournir des informations et/ou pour donner des recommandations relatives aux mesures qu'il convient de prendre lors de l'utilisation, de la modification, de la mise à disposition ou du retour du dispositif médical).

Ces définitions sont en cours de révision au niveau européen et seront probablement remplacées par les notions de Field Safety Corrective Action (FSCA) et Field Safety Notice, lesquelles 
ont une signification semblable mais non identique.

\section{Définitions américaines}

Au contraire du système européen, les Etats-Unis classent les rappels en trois catégories (Code of Federal Regulations 21, Part 7: Enforcement policy):

Recall means a firm's removal or correction of a marketed product that the Food and Drug Administration considers to be in violation of the laws it administers and against which the agency would initiate legal action, e.g., seizure. Recall does not include a market withdrawal or a stock recovery. (1) Class I is a situation in which there is a reasonable probability that the use of, or exposure to, a violative product will cause serious adverse health consequences or death. (2) Class II is a situation in which use of, or exposure to, a violative product may cause temporary or medically reversible adverse health consequences or where the probability of serious adverse health consequences is remote. (3) Class III is a situation in which use of, or exposure to, a violative product is not likely to cause adverse health consequences.

\section{Canaux d'information et devoir de communiquer}

Lors de rappels, chaque participant est tenu d'informer l'organe qui le suit dans la chaîne de distribution du dispositif. Swissmedic contrôle si la procédure est appliquée correctement du fabricant au centre d'implantation (information aux centres d'implantation). Les directions cantonales de la santé publique peuvent en tout temps contrôler l'ultime démarche (du médecin au patient).

Dans notre cas, le fabricant informe le président à l'attention du groupe de travail. Le président se charge de télécharger l'information sur le site internet du groupe de travail (www.pace maker.ch) et de l'envoyer immédiatement par courrier électronique à tous les membres du groupe. De surcroît, le fabricant remet au président la liste complète de tous les implants concernés en Suisse.

La firme responsable de l'implant informe par écrit tous les centres d'implantation concernés par lettre recommandée ou par un autre moyen approprié. Elle leur fournit également la liste des dispositifs qu'ils ont implantés.

Le centre d'implantation est responsable de vérifier que la liste d'attribution des implants aux patients concernés est correcte. La responsabilité de la transmission correcte du rappel incombe au centre ayant pratiqué l'implantation. Celui-ci est tenu d'identifier les patients concernés dont le suivi est assumé par un autre centre et d'informer ce dernier le plus rapidement possible de manière à ce qu'il puisse transmettre l'information au patient.

Le centre chargé du suivi doit immédiatement informer les patients par écrit et leur proposer une date appropriée pour un entretien détaillé et adapté à la situation. Ce centre est également tenu d'envoyer aux médecins de famille une copie de la lettre adressée aux patients, accompagnée d'une copie de la lettre d'information du fabricant. Il doit veiller à ce que son service des urgences soit informé des données spécifiques propres aux patients équipés d'implants potentiellement défectueux et puisse en tirer les justes conséquences. Selon la situation, il convient de joindre à l'attestation accompagnant le stimulateur cardiaque ou contenant le code CIM une notice donnant l'information nécessaire.

\section{Mesures médicales}

Il incombe au centre chargé du suivi de prendre les mesures médicales nécessaires sur une base individuelle, d'entente avec le patient et en tenant compte des recommandations du fabricant et des sociétés spécialisées nationales ou internationales. Le centre doit disposer des bases décisionnelles et informations importantes en la matière. En cas de dysfonctionnement potentiellement dangereux d'implants, les décisions prises et les traitements appliqués pour chaque patient doivent être consignés dans le dossier médical et sur une liste séparée.

On devrait tenir compte du fait que l'incidence d'un dysfonctionnement peut changer au cours du temps et qu'il convient donc de réévaluer les mesures prises et, en particulier, l'opportunité d'un changement électif éventuel de l'implant. Si la situation change, le fabricant informe des conséquences qui en découlent sur le plan de la sécurité. Il incombe aux centres d'implantation et de suivi de transmettre ces informations aux patients et à leurs médecins de famille.

\section{Indemnisation des coûts}

Sur le plan juridique, on ne sait pas précisément qui, du fabricant de l'implant, des autres responsables civils potentiels (hôpital, médecins, Swissmedic) ou des assureurs, doit prendre en charge les coûts occasionnés par les mesures visant à pallier un dysfonctionnement potentiel d'implants.

Les fabricants prennent en charge les coûts consécutifs à l'information du patient au sujet d'un rappel effectué pour des raisons de sécurité. Par là, on entend l'entretien avec le patient ainsi que les mesures recommandées dans la fiche d'avertissement du dit rappel. Si ce rappel induit des contrôles supplémentaires allant au-delà des 
recommandations données par le fabricant dans le mode d'emploi, les coûts sont également pris en charge par les firmes.

Lorsque les coûts occasionnés par des interventions chirurgicales et des séjours hospitaliers stationnaires ou ambulatoires sont consécutifs à un rappel, il conviendrait de régler la question de la prise en charge avant l'intervention entre les répondants des coûts et le fabricant ou d'autres tiers éventuellement impliqués. Cette réglementation comprend aussi les coûts de l'implant remplacé, sous réserve de la garantie du produit et de la responsabilité civile du fabricant. Les patients concernés doivent être informés des coûts consécutifs à un dysfonctionnement potentiel d'implants lors de l'entretien d'information. Le fournisseur de prestations doit en outre attirer l'attention du répondant des coûts sur l'existence possible d'une garantie dans le cadre de la responsabilité civile du fabricant.

\section{Aspects épidémiologiques et de médecine légale}

Dans les faits, il est très probable que le taux de dysfonctionnements soit plus élevé qu'on ne le suppose, car aucun contrôle systématique des implants n'est effectué post mortem. La détermination aussi exacte que possible de l'incidence des dysfonctionnements est toutefois très importante pour détecter une mise en danger éventuelle des patients.

Par conséquent, les mesures suivantes devraient être prises à chaque rappel:

a) Mesures rétroactives: les centres qui assument le suivi sont invités à effectuer des investigations dans le cas de personnes déjà décédées porteuses d'un implant concerné par un rappel consécutif à un dysfonctionnement potentiellement dangereux, pour voir si une défectuosité de l'appareil pourrait être la cause du décès. En cas de soupçon fondé, les centres doivent en informer Swissmedic et le président du groupe de travail.

b) Mesures à compter de la date de rappel: si le porteur d'un implant concerné par un rappel meurt subitement et qu'un dysfonctionnement pourrait en être la cause, il faut si possible examiner l'implant. Dans une telle situation, le décès doit être annoncé comme inhabituel au juge d'instruction. Nous recommandons de remettre l'implant à des experts du groupe de travail qui n'ont pas été impliqués dans l'assistance médicale du patient décédé. Ces experts examinent l'implant et communiquent en l'espace de deux jours ouvrables au donneur d'ordre et au fabricant si l'examen effectué avec un appareil de programmation permet d'exclure une défectuosité; ils établissent la documentation nécessaire et informent Swissmedic.

\section{Appendice}

Ont participé à la mise au point du commentaire: - membres du comité du groupe de travail Stimulation cardiaque et électrophysiologie de la Société suisse de cardiologie: Dr Jürg Fuhrer, Berne (planification, présidence et manuscrit); M. Istvan Babotai, dr en sciences techniques, Zurich; Dr Urs Bauersfeld, privatdocent, Zurich; Dr Jean-Luc Crevoisier, Delémont; Dr Hans Gloor, Aarau; Prof. Stefan Osswald, Bâle; Dr Jürg Schläpfer, privat-docent, Lausanne; Dr Juan Sztajzel, privat-docent, Genève;

- représentant de la Société suisse de médecine légale: Prof. Ulrich Zollinger, Berne;

- représentant de l'autorité délivrant les autorisations (Swissmedic): M. Andrea Sparti, dr en sciences naturelles, Berne;

- représentant de la Fédération des associations suisses du commerce et de l'industrie de la technologie médicale (FASMED): M. Jürg $\mathrm{H}$. Schnetzer, avocat;

- représentants de l'industrie (délégués des firmes sans droit de vote, présents dans le groupe de travail Stimulation cardiaque et électrophysiologie): M. Sylvain Amiguet (Biotronik); Mme Daniela Amstutz (Vitatron); M. Martin Deola (Medtronic); M. Hans-Peter Schefer (Guidant); M. Bruno Sidler (St. Jude Medical); M. Lotfi Youssef (Sorin Group).

Assument la responsabilité du commentaire:

- Société suisse de cardiologie (SSC), représentée par: Dr A. Jaussi, président;

- santésuisse, association faîtière de la branche de l'assurance-maladie sociale, représentée par: M. Marc-André Giger, directeur; Dr Reto Guetg, médecin-conseil; M. Andreas Altermatt, lic. en droit, avocat et notaire;

- service juridique de la Fédération des médecins suisses (FMH), représenté par: M. Hanspeter Kuhn, avocat, secrétaire général adjoint;

- Dachverband Schweizerischer Patientenstellen (DVSP), représenté par: Mme Erika Ziltener, présidente. 